\title{
RESONANT FREQUENCIES IN AN ELECTROMAGNETIC ECCENTRIC SPHERICAL CAVITY*
}

\author{
BY \\ JOHN D. KANELLOPOULOS AND JOHN G. FIKIORIS \\ National Technical University of A thens
}

\begin{abstract}
The interior boundary-value electromagnetic (vector) problem in the region between two perfectly conducting spheres of radii $R_{1}, R_{2}$ and distance $d$ between their centers is considered. Surface singular integral equations are used to formulate the problem. Use of spherical vector wave functions and related addition theorems reduces the solution of the integral equations to the problem of solving an infinite set of linear equations. Their determinant is evaluated in powers of $k d=2 \pi d / \lambda$ to a few terms. It is then specialized to the axially symmetric case and set equal to zero. This yields closed-form expressions for the coefficients $g_{n s}$ in the resulting relations $\omega_{n s}(k d)=\omega_{n s}(0)\left[1+g_{n s}(k d)^{2}\right.$ $+\cdots]$ for the natural frequencies of the cavity. Numerical results, comparisons and possible generalizations are also included.
\end{abstract}

Introduction. The interior boundary-value acoustic problem in the region between two spheres of radii $R_{1}, R_{2}$ and distance $d$ between their centers (see Fig. 1) for both Dirichlet and Neumann boundary conditions, has been solved elsewhere [1]. The present paper deals with the corresponding electromagnetic problem between perfectly conducting eccentric spheres and should be read in conjuction with [1]. The motivation for considering analytical and exact solutions to such problems, their advantages over numerical ones and the possible generalizations to other shapes are discussed in [1] and will not be repeated here. An updated and extensive reference list may also be found in [1]. However, the significant differences between acoustic and electromagnetic cavities that will be brought to light in this paper require a separate treatment.

As in [1], let small letters $\left(p_{1}, q_{2}\right)$ denote points on $S_{1}$ and $S_{2}$ and capitals $(P, Q)$ points not on $S_{1}, S_{2}$. A fixed point on $S_{1}$ or $S_{2}$ is designated by $p_{1}\left(\theta_{1}, \phi_{1}\right)$ or $p_{2}\left(\theta_{2} \nabla, \phi_{2}\right)$, a variable one by $q_{1}\left(\theta_{1}^{\prime}, \phi_{1}^{\prime}\right)$ or $q_{2}\left(\theta_{2}^{\prime}, \phi_{2}^{\prime}\right)$, with the primes indicating, in particular, variables of integration. Under this notation $R_{1}, \theta_{1}, \phi_{1}$ (or $R_{2}, \theta_{2}^{\prime}, \phi_{2}^{\prime}$ ) indicate spherical coordinates of $p_{1}$ (or $q_{2}$ ) with respect to centers $O_{1}\left(\right.$ or $\left.O_{2}\right)$ of the surface $S_{1}\left(\right.$ or $\left.S_{2}\right)$ to which the point belongs. However, $p_{1}\left(R_{12}, \theta_{12}, \phi_{12}\right)$ or $q_{2}\left(R_{21}^{\prime}, \theta_{21}^{\prime}, \phi_{21}^{\prime}\right)$ are spherical coordinates of the same points with respect to the other center, $O_{2}$ or $O_{1}$, indicated by the second subscript. Normal unit vectors $\hat{n}_{p 1}, \hat{n}_{q 2}$ on $S_{1}, S_{2}$ are directed out of $V$, as shown in Fig. 1 .

The electromagnetic cavity problem in $V$, with perfectly conducting boundaries $S_{1}$ and

* Received December 19, 1977. Partially supported by the National Research Foundation of Greece. The authors wish to thank Dr. E. Lecatsas for his assistance in the numerical evaluation of the $g_{v s}$. 
$S_{2}$, can be formulated in terms of homogeneous surface integral equations on $S_{1}, S_{2}[2,3$, $4]$ for the unknown surface current densities $j_{1}\left(p_{1}\right), \bar{j}_{2}\left(p_{2}\right)$ :

$$
\begin{aligned}
& -2 \pi \bar{j}_{1}\left(p_{1}\right)=\hat{n}_{p 1} \times \int_{S_{1}}\left[\bar{j}_{1}\left(q_{1}\right) \times \nabla^{\prime} G\left(p_{1}, q_{1}\right)\right] d S_{q 1} \\
& +\hat{n}_{p 1} \times \int_{S_{2}}\left[\bar{j}_{2}\left(q_{2}\right) \times \nabla^{\prime} G\left(p_{1}, q_{2}\right)\right] d S_{q 2}, \\
& -2 \pi \bar{j}_{2}\left(p_{2}\right)=\hat{n}_{p 2} \times \int_{S_{1}}\left[\bar{j}_{1}\left(q_{1}\right) \times \nabla^{\prime} G\left(p_{2}, q_{1}\right)\right] d S_{q 1} \\
& +\hat{n}_{p 2} \times \int_{S_{2}}\left[\bar{j}_{2}\left(q_{2}\right) \times \nabla^{\prime} G\left(p_{2}, q_{2}\right)\right] d S_{q 2},
\end{aligned}
$$

where

$$
G(P, Q)=G(R)=\exp (i k R) / R \quad(R=P Q)
$$

is the free space Green's function and $\nabla^{\prime}$ operates on the prime coordinates of the point $q_{1}$ (or $\left.q_{2}\right)$. The integrals involving $\nabla^{\prime} G\left(p_{1}, q_{1}\right)$ or $\nabla^{\prime} G\left(p_{2}, q_{2}\right)$ are convergent singular surface integrals $[2,4,5]$.

In the following, analytical solutions of the preceding integral equations are obtained, after properly evaluating the singular integrals by a convenient limiting process. Use also is made of translational addition theorems for spherical vector wave functions [6]. In the limit of small $k d$ and in the particular but important case of axially symmetric fields, an exact evaluation of the elements of an infinite determinant and of the determinant itself is achieved that yields the values of the resonant frequencies $\omega_{\nu s}(k d)$ to second order in $k d$, in exact analogy with the methods of [1].

Solution of the integral equations. The unknown surface current densities $j_{1}\left(q_{1}\right)$, $\bar{j}_{2}\left(q_{2}\right)$, being tangential to the surfaces $S_{1}$ and $S_{2}$ of the spheres, may be conveniently expanded in terms of a complete set of complex spherical surface vector functions $\bar{B}, \bar{C}$ of the angles $\theta, \phi[7]$. The latter are directly related to the complex spherical eigenvectors $\dot{m}$, $\bar{n}, \bar{l}$ by the definitions:

$$
\begin{aligned}
\dot{m}_{m n}(r, \theta, \phi)=[n(n+1)]^{1 / 2} z_{n}(k r) \bar{C}_{n m}(\theta, \phi) & =z_{n}(k r) \exp (i m \phi)\left[\frac{i m}{\sin \theta} P_{n}^{m}(\cos \theta) \hat{\theta}-\frac{\partial P_{n}^{m}}{\partial \theta} \hat{\phi}\right], \\
\bar{n}_{m n}(r, \theta, \phi)= & n(n+1) \frac{z_{n}(k r)}{k r} \bar{P}_{m n}(\theta, \phi)+[n(n+1)]^{1 / 2} \frac{z_{n}^{d}(k r)}{k r} \bar{B}_{m n}(\theta, \phi) \\
= & \frac{\exp (i m \phi)}{k r}\left\{n(n+1) z_{n}(k r) P_{n}^{m}(\cos \theta) \hat{r}+z_{n}^{d}(k r)\right. \\
& \left.\cdot\left[\frac{\partial P_{n}^{m}}{\partial \theta} \hat{\theta}+\frac{i m}{\sin \theta} P_{n}^{m}(\cos \theta) \hat{\phi}\right]\right\},
\end{aligned}
$$

where $m=-n,-n+1, \cdots 0,1, \cdots n, z_{n}(x)$ is the general spherical Bessel function and $z_{n}^{d}(x)=\left[x z_{n}(x)\right]^{\prime}$. In this notation, similar to Cruzan's [6], $P_{n}^{-m}(x)(m \geq 0)$ is defined by

$$
P_{n}^{-m}(x)=(-1)^{m} \frac{(n-m) !}{(n+m) !} P_{n}^{m}(x)
$$


differing by a $(-1)^{m}$ factor from another common definition [7, 8]. Anyone of the above complex vectors ( $\bar{m}, \bar{n}, \bar{B}, \bar{C}$ etc.) is related to the corresponding even and odd real vectors, defined in $[7,8]$ for $m \geq 0$, as follows:

$$
\bar{C}_{m n}=\bar{C}_{m n}^{e}+i \bar{C}_{m n}^{0}, \bar{C}_{-m n}=(-1)^{m} \frac{(n-m) !}{(n+m) !}\left[\bar{C}_{m n}^{e}-i \bar{C}_{m n}^{2}\right], \quad(m \geq 0),
$$

where $\bar{m}_{m n}, \bar{n}_{m n}, \bar{B}_{m n}$ etc. may be substituted for $\bar{C}_{m n}$. Relations among the surface vectors $\bar{P}, \bar{B}, \bar{C}$ and their orthogonal properties are as follows:

$$
\begin{gathered}
\hat{r} \times \bar{P}_{m n}=0, \hat{r} \times \bar{B}_{m n}=-\bar{C}_{m n}, \hat{r} \times \bar{C}_{m n}=\bar{B}_{m n} ; \\
\bar{P}_{m n} \cdot \bar{B}_{m^{\prime} n^{\prime}}=\bar{P}_{m n} \cdot \bar{C}_{m^{\prime} n^{\prime}}=\bar{B}_{m n} \cdot \bar{C}_{m n}=0 ; \\
\iint \bar{P}_{m n} \cdot \bar{P}_{m n} d \Omega=\iint \bar{B}_{m n} \cdot \bar{B}_{m n} d \Omega=\iint \bar{C}_{m n} \cdot \bar{C}_{m n} d \Omega \\
=\iint \bar{B}_{m n} \cdot \bar{C}_{m^{\prime} n^{\prime}} d \Omega=0 ; \\
\iint \bar{P}_{m n} \cdot \bar{P}_{-m^{\prime}, n^{\prime}} d \Omega=\iint \bar{B}_{m n} \cdot \bar{B}_{-m^{\prime}, n^{\prime}} d \Omega=\iint \bar{C}_{m n} \cdot \bar{C}_{-m^{\prime}, n^{\prime}} d \Omega \\
=\frac{4 \pi}{2 n+1}(-1)^{m} \delta_{m m^{\prime}} \delta_{n n^{\prime}},
\end{gathered}
$$

where $d \Omega=\sin \theta d \theta d \phi$ and the integration is over the ranges $0 \leq \theta \leq \pi, 0 \leq \phi \leq 2 \pi ; n, n^{\prime}$ $=1,2, \cdots ; m=-n,-n+1, \cdots 0,1, \cdots n ; m^{\prime}=-n^{\prime}+1, \cdots 0,1, \cdots n^{\prime} ; \delta_{n n^{\prime}}=1$ for $n$ $=n^{\prime}$ and $\delta_{n n^{\prime}}=0$ for $n \neq n^{\prime}$.

In expanding now $\bar{j}_{1}\left(q_{1}\right)$ and $\bar{j}_{2}\left(q_{2}\right)$ no loss of generality occurs if only one value of the index $m$, namely $m=M$, is considered. As in [1] this is due basically to the fact that with $O_{1} O_{2}$ along the $z$ axis the azimuthal angles remain the same $\left(\phi_{1}^{\prime}=\phi_{12}^{\prime}, \phi_{2}^{\prime}=\phi_{21}^{\prime}\right)$ when referred to $O_{1}$ or $O_{2}$. Therefore:

$$
\begin{aligned}
& \bar{j}_{1}\left(q_{1}\right)=\sum_{s=|M|}^{\infty}[s(s+1)]^{1 / 2}\left[\frac{A_{M s}}{j_{s}^{d}\left(x_{1}\right)} \bar{B}_{M s}\left(\theta_{1}^{\prime}, \phi_{1}^{\prime}\right)+\frac{F_{M s}}{x_{1} j_{s}\left(x_{1}\right)} \bar{C}_{M S}\left(\theta_{1}^{\prime}, \phi_{1}^{\prime}\right)\right], \\
& \dot{j}_{2}\left(q_{2}\right)=\sum_{s=|M|}^{\infty}[s(s+1)]^{1 / 2}\left[\frac{G_{M s}}{j_{s}^{d}\left(x_{2}\right)} \bar{B}_{M s}\left(\theta_{2}^{\prime}, \phi_{2}^{\prime}\right)+\frac{H_{M s}}{x_{2} j_{s}\left(x_{2}\right)} \bar{C}_{M s}\left(\theta_{2}^{\prime}, \phi_{2}^{\prime}\right)\right],
\end{aligned}
$$

where $x_{1}=k R_{1}, x_{2}=k R_{2}$. The more general case would simply involve a superposition (or further summation over $M=-s,-s+1, \cdots 0,1, \cdots s$ ).

Use will also be made of the well-known expansion [7]:

$$
\begin{aligned}
& \overline{\bar{G}}(P, Q)=\overline{\bar{I}} \frac{\exp (i k R)}{R}=i k \sum_{n=0}^{\infty} \frac{2 n+1}{n(n+1)} \sum_{m=-n}^{\infty}(-n)^{m}\left[\bar{m}_{m, n}^{(1)}(Q) \bar{m}_{m n}^{(3)}(P)\right. \\
& +\bar{n}_{-m, n}^{(1)}(\mathrm{Q}) \bar{n}_{m n}^{(3)}(P)+n(n+1)\left[_{-m, n}^{(1)}(Q) l_{m n}^{(3)}(P)\right], \quad\left(r=O P>r^{\prime}=O Q\right)
\end{aligned}
$$

for the free space Green's dyadic in terms of the complex spherical eigenvectors. The superscript (1) is associated with $j_{n}(x)$, while (3) implies use of $h_{n}^{(1)}(x) \equiv h_{n}(x)$. On the basis of (7), written for the $\bar{m}, \bar{n}, \bar{l}$ vectors, it is easy to show that (13) is equivalent to the alternative formula involving the even and odd vectors [7]. In (13) $\bar{I}$ is the identity dyadic $(\bar{A} \cdot \bar{I}=\bar{I} \cdot \bar{A}=\bar{A})$ and in case $r(=O P)<r^{\prime}(=O Q)$ one simply interchanges the 
superscripts 1 and 3 of the spherical eigenvectors, or, equivalently, their arguments $P$ and $Q$.

In evaluating the surface integrals of (1) and (2) it is convenient to consider initially the integral

$$
\begin{aligned}
\bar{L}(P) & =\nabla \times \int_{S} \bar{j}(q) G(P, q) d S_{q}=\nabla \times \int_{S} \bar{j}(q) \cdot \bar{G}(P, q) d S_{q} \\
& =\int_{S} \bar{j}(q) \times \nabla^{\prime} G(P, q) d S_{q},
\end{aligned}
$$

where $S$ is a spherical surface of radius $a$ and center $O, q\left(a, \theta^{\prime}, \phi^{\prime}\right)$ is a point on $S, P(r, \theta, \phi)$ is a fixed point not on $S(r>a$ or $r<a), R=q P$ and $j(q)$ is a vector tangential to $S$, which can be expanded in a series like (11) with the subscript 1 deleted $(x=k a)$. Under these conditions it is well known $[2,5]$ that $\bar{L}(P)$ is an analytic vector function of $P(r, \theta, \phi)$. The last expression for $\bar{L}(P)$ can be deduced from the first and the relation $\nabla \times[\bar{j}(q) G(P, q)]=$ $-\bar{j}(q) \times \nabla G=\bar{j}(q) \times \nabla^{\prime} G$.

Substitution of (13) into (14) and term-by-term integration yields, in case $r>a$, terms of the form

$$
\bar{m}_{m n}^{(3)}(P) \int_{S} \bar{j}(q) \cdot \bar{m}_{m, n}^{(1)}(q) d \Omega_{q}, \quad \text { where } d S_{q}=a^{2} d \Omega_{q} .
$$

For $r<a$ the superscripts of the eigenvectors are interchanged. Noticing that $\nabla$ operates on $r, \theta, \phi$ of $P$, that $\nabla \times\left[=0, \nabla \times \bar{m}=k \bar{n}, \nabla \times \bar{n}=k \bar{m}\right.$ and that $\bar{j} \cdot \bar{P}_{m n}=0(\bar{j}$ being transverse and $\bar{P}$ radial), one finds

$$
\begin{aligned}
\bar{L}(P)=i x^{2} \sum_{n=0}^{\infty} \frac{2 n+1}{[n(n+1)]^{1 / 2}} \sum_{m=-n}^{n}(-1)^{m}\left[\bar{n}_{m n}^{(3)}(P) j_{n}(x) \int_{S} \bar{j}\left(\theta^{\prime}, \phi^{\prime}\right) \cdot \bar{C}_{-m, n}\left(\theta^{\prime}, \phi^{\prime}\right) d \Omega_{q}\right. \\
\left.+\bar{m}_{m n}^{(3)}(P) \frac{j_{n}^{d}(x)}{x} \int_{S} \bar{j}\left(\theta^{\prime}, \phi^{\prime}\right) \cdot \bar{B}_{-m, n}\left(\theta^{\prime}, \phi^{\prime}\right) d \Omega_{q}\right],
\end{aligned}
$$

where $\bar{m}_{m, n}^{(1)}(q)$ and $\bar{n}_{m, n}^{(1)}(q)$ have been replaced in accordance with (4), (5). Substitution of (11) (with the subscript 1 deleted) for $\bar{j}(q)$ in (15) and use of the orthogonal properties (9), (10) yield:

$$
\bar{L}(P)=4 \pi i x \sum_{s=|M|}^{\infty}\left[F_{M s} \bar{n}_{M s}^{(3)}(P)+A_{M s} \bar{m}_{M s}^{(3)}(P)\right], \quad r>a .
$$

For $r<a$ a similar procedure yields:

$$
\bar{L}(P)=4 \pi i x \sum_{s=|\mathcal{M}|}^{\infty}\left[F_{M s} \frac{h_{s}(x)}{j_{s}(x)} \bar{n}_{M s}^{(1)}(P)+A_{M s} \frac{h_{s}^{d}(x)}{j_{s}^{d}(x)} \dot{m}_{M s}^{(1)}(P)\right] .
$$

On the basis of (17) and (16) it is now possible to evaluate immediately the nonsingular integrals in (1) and (2), respectively:

$$
\begin{array}{r}
\hat{n}_{p 1} \times \int_{S 2}\left[\overline{j_{2}}\left(q_{2}\right) \times \nabla^{\prime} G\left(p_{1}, q_{2}\right)\right] d S_{q 2}=4 \pi i x_{2} \hat{n}_{p 1} \times \sum_{n=|M|}^{\infty}\left[H_{M n} \frac{h_{n}\left(x_{2}\right)}{j_{n}\left(x_{2}\right)} \bar{n}_{M n}^{(1)}\left(R_{12}, \theta_{12}, \phi_{12}\right)\right. \\
\left.+G_{M n} \frac{h_{n}^{d}\left(x_{2}\right)}{j_{n}^{d}\left(x_{2}\right)} \bar{m}_{M n}^{(1)}\left(R_{12}, \theta_{12}, \phi_{12}\right)\right],
\end{array}
$$


where (12) was used instead of (11) and the coordinates of $p_{1}\left(R_{12}, \theta_{12}, \phi_{12}\right)$ with respect to center $\mathrm{O}_{2}$ have been explicitly shown. Also:

$$
\begin{gathered}
\hat{n}_{p 2} \times \int_{S 1}\left[\bar{V}_{1}\left(q_{1}\right) \times \nabla^{\prime} G\left(p_{2}, q_{1}\right)\right] d S_{q 1}=4 \pi i x_{1} \hat{n}_{p 2} \times \sum_{n=|M|}^{\infty}\left[F_{M n} \dot{n}_{M n}^{(3)}\left(R_{21}, \theta_{21}, \phi_{21}\right)\right. \\
\left.+A_{M n} \dot{m}_{M n}^{(3)}\left(R_{21}, \theta_{21}, \phi_{21}\right)\right] .
\end{gathered}
$$

In order to evaluate the singular surface integrals in (1), (2) it is convenient to return to (14) and cross-multiply it by $\hat{n}_{p}=\hat{r}_{p}$, where $p(a, \theta, \phi)$ is a point on $S$, on the same radius as $P(R, \theta, \phi)$, with $r>a$ or $r<a$. It is then possible to make use of the classical result $[2,4,5]$ :

$$
\hat{r}_{p} \times \int_{S}\left[\tilde{j}(q) \times \nabla^{\prime} G(p, q)\right] d S_{q}=\lim _{P \rightarrow p}\left[\hat{r}_{p} \times \bar{L}(P)\right] \mp 2 \pi j(p),
$$

in which the upper/lower sign of the last term corresponds to $P$ approaching $p$ from the exterior/interior of $S$, i.e., for $r>a / r<a$. Before the limit is taken, it is possible, as before, to use (16) (for $r>a$ ) or (17) (for $r<a$ ) to obtain

$\hat{r}_{p} \times \int_{S}\left[j(q) \times \nabla^{\prime} G(p, q)\right] d S_{q}=-2 \pi j(p)+4 \pi i x \lim _{P \rightarrow p} \sum_{s=|M|}^{\infty}\left[F_{M s} \hat{r}_{p} \times \tilde{n}_{M s}^{(3)}(P)\right.$

$$
\begin{aligned}
= & -2 \pi j(p)+4 \pi i x \sum_{s=|M|}^{\infty}[s(s+1)]^{1 / 2} \\
& \cdot\left[-F_{M s} \frac{h_{s}^{d}(x)}{x} \bar{C}_{M s}(\theta, \phi)+A_{M s} h_{s}(x) \bar{B}_{M s}(\theta, \phi)\right]
\end{aligned}
$$

or

$$
\begin{aligned}
\hat{r}_{p} \times \int_{s}\left[\tilde{j}(q) \times \nabla^{\prime} G(p, q)\right] d S_{q}= & +2 \pi j(p)+4 \pi i x \lim _{P \rightarrow p} \sum_{s=|M|}^{\infty}\left[F_{M s} \frac{h_{s}(x)}{j_{s}(x)}\right. \\
& \left.\cdot \hat{r}_{p} \times \bar{n}_{M s}^{-11}(P)+A_{M s} \frac{h_{s}^{d}(x)}{j_{s}^{d}(x)} \hat{r}_{p} \times \bar{m}_{M s}^{(1)}(P)\right] \\
= & 2 \pi j(p)+4 \pi i x \sum_{s=|M|}^{\infty}[s(s+1)]^{1 / 2}\left[-F_{M s} \frac{h_{s}(x) j_{s}^{d}(x)}{x j_{s}(x)}\right. \\
& \left.\cdot \bar{C}_{M s}(\theta, \phi)+A_{M s} \frac{h_{s}^{d}(x)}{j_{s}^{d}(x)} j_{s}(x) \bar{B}_{M s}(\theta, \phi)\right],
\end{aligned}
$$

in which (4), (5) and (8) were also used. The two results for the singular surface integral are identical, if the relations

$$
\begin{aligned}
\bar{j}(p) & =\sum_{s=|M|}^{\infty}[s(s+1)]^{1 / 2}\left[\frac{A_{M s}}{j_{s}^{d}(x)} \bar{B}_{M s}(\theta, \phi)+\frac{F_{M s}}{x j_{s}(x)} \bar{C}_{M s}(\theta, \phi)\right], \\
\frac{i}{x} & =j_{s}(x) h_{s}^{d}(x)-h_{s}(x) j_{s}^{d}(x)
\end{aligned}
$$

are also invoked. 
It is now possible to substitute back into the integral equations (1) and (2). Recalling from Fig. 1 that $\hat{n}_{p_{1}}=-\hat{r}_{p_{1}}, \hat{n}_{p_{2}}=\hat{r}_{p_{2}}$, it is convenient to use (22) in (1) and (21) in (2). Together with (18), (19) and (12) instead of (11), wherever $\hat{j}_{2}\left(q_{2}\right)$ is involved, one finally gets:

$$
\begin{gathered}
x_{1} \sum_{s=|M|}^{\infty}[s(s+1)]^{1 / 2}\left[-F_{M s} \frac{h_{s}\left(x_{1}\right) j_{s}^{d}\left(x_{1}\right)}{x_{1} j_{s}\left(x_{1}\right)} \bar{C}_{M s}\left(\theta_{1}, \phi_{1}\right)+A_{M s} \frac{h_{s}^{d}\left(x_{1}\right)}{j_{s}^{d}\left(x_{1}\right)} j_{s}\left(x_{1}\right) \bar{B}_{M s}\left(\theta_{1}, \phi_{1}\right)\right]+x_{2} \hat{r}_{p_{1}} \\
\times \sum_{n=|M|}^{\infty}\left[H_{M n} \frac{h_{n}\left(x_{2}\right)}{j_{n}\left(x_{2}\right)} \tilde{n}_{M n}^{(1)}\left(R_{12}, \theta_{12}, \phi_{12}\right)+G_{M n} \frac{h_{n}^{d}\left(x_{2}\right)}{j_{n}^{d}\left(x_{2}\right)} \bar{m}_{M n}^{(1)}\left(R_{12}, \theta_{12}, \phi_{12}\right)\right]=0 \\
x_{1} \hat{r}_{p_{2}} \times \sum_{n=|M|}^{\infty}\left[F_{M n} \bar{n}_{M n}^{(3)}\left(R_{21}, \theta_{21}, \phi_{21}\right)+A_{M n} \bar{m}_{M n}^{(3)}\left(R_{21}, \theta_{21}, \phi_{21}\right)\right]+\sum_{s=|M|}^{\infty}[s(s+1)]^{1 / 2} \\
{\left[-H_{M s} h_{s}^{d}\left(x_{2}\right) \bar{C}_{M s}\left(\theta_{2}, \phi_{2}\right)+G_{M s} x_{2} h_{s}\left(x_{2}\right) \bar{B}_{M s}\left(\theta_{2}, \phi_{2}\right)\right]=0}
\end{gathered}
$$

The second/first term in $(23) /(24)$ contains spherical eigenvectors with respect to origin $O_{2} / O_{1}$. They may be expanded into sums of spherical eigenvectors with respect to $O_{1} / O_{2}$ (like the other term of the equation) using the well-known translation al addition theorems of Cruzan [6]:

$$
\begin{aligned}
& \bar{m}_{M n}^{(1)}\left(R_{12}, \theta_{12}, \phi_{12}\right)=\sum_{s=1}^{\infty} \sum_{\mu=-s}^{s}\left[A_{\mu s}^{M n} \bar{m}_{\mu s}^{(1)}\left(R_{1}, \theta_{1}, \phi_{1}\right)+B_{\mu s}^{M n} \bar{n}_{\mu s}^{(1)}\left(R_{1}, \theta_{1}, \phi_{1}\right)\right], \\
& \tilde{n}_{M n}^{(1)}\left(R_{12}, \theta_{12}, \phi_{12}\right)=\sum_{s=1}^{\infty} \sum_{\mu=-s}^{s}\left[A_{\mu s}^{M n} \bar{n}_{\mu s}^{(1)}\left(R_{1}, \theta_{1}, \phi_{1}\right)+B_{\mu s}^{M n} \bar{m}_{\mu s}^{(1)}\left(R_{1}, \theta_{1}, \phi_{1}\right)\right],
\end{aligned}
$$

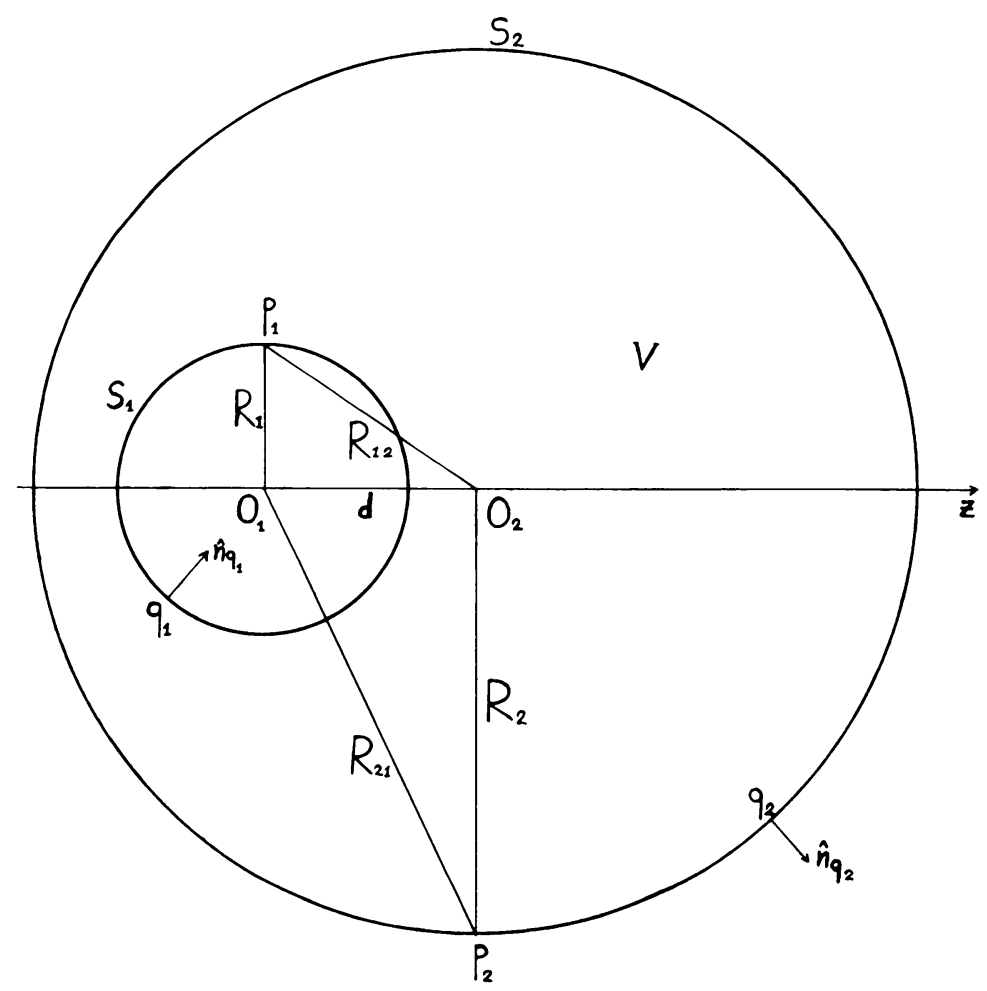

FIG. 1. The geometry of the cavity. 


$$
\begin{aligned}
\bar{m}_{M n}^{(3)}\left(R_{21}, \theta_{21}, \phi_{21}\right)= & \sum_{s=1}^{\infty} \sum_{\mu=-s}^{s}\left[C_{\mu s}^{M n} \bar{m}_{\mu s}^{(3)}\left(R_{2}, \theta_{2}, \phi_{2}\right)\right. \\
& \left.+D_{\mu s}^{M n} \bar{n}_{\mu s}^{(3)}\left(R_{2}, \theta_{2}, \phi_{2}\right)\right], \quad\left(R_{2}>d\right), \\
\bar{n}_{M n}^{(3)}\left(R_{21}, \theta_{21}, \phi_{21}\right)= & \sum_{s=1}^{\infty} \sum_{\mu=-s}^{s}\left[C_{\mu s}^{M n} \bar{n}_{\mu s}^{(3)}\left(R_{2}, \theta_{2}, \phi_{2}\right)\right. \\
& \left.+D_{\mu s}^{M n} \bar{m}_{\mu s}^{(3)}\left(R_{2}, \theta_{2}, \phi_{2}\right)\right], \quad\left(R_{2}>d\right),
\end{aligned}
$$

with

$$
\begin{aligned}
A_{\mu s}^{M n}= & (-1)^{\mu} \sum_{p} a(M, n|-\mu, s| p) a(n, s, p) j_{p}(k d) P_{p}^{M-\mu}\left(\cos \theta_{0}\right) \exp \left(i(M-\mu) \phi_{0}\right), \\
B_{\mu s}^{M n}= & (-1)^{\mu} \sum_{p} a(M, n|-\mu, s| p+1, p) b(n, s, p+1) j_{p+1}(k d) P_{p+1}^{M-\mu}\left(\cos \theta_{0}\right) \\
& \cdot \exp \left(i(M-\mu) \phi_{0}\right), \\
C_{\mu s}^{M n}= & (-1)^{\mu} \sum_{p} a(M, n|-\mu, s| p) a(n, s, p) j_{p}(k d) P_{p}^{M-\mu}\left(\cos \theta_{0}^{\prime}\right) \exp \left(i(M-\mu) \phi_{0}^{\prime}\right) \\
D_{\mu s}^{M n}= & (-1)^{\mu} \sum_{p} a(M, n|-\mu, s| p+1, p) b(n, s, p+1) j_{p+1}(k d) P_{p+1}^{M-\mu}\left(\cos \theta_{0}^{\prime}\right) \\
& \cdot \exp \left(i(M-\mu) \phi_{0}^{\prime}\right),
\end{aligned}
$$

where $d, \theta_{0}, \phi_{0}$ are the coordinates of $O_{1}$ with respect to $O_{2}, d, \theta_{0}^{\prime}, \phi_{0}^{\prime}$ those of $O_{2}$ with respect to $O_{1}$ and the summation index $p$ varies from $|n-s|$ to $n+s$ by steps of 2 . Finally, the symbols $a(M, n|-\mu, s| p), a(M, n|-\mu, s| p+1, p), a(n, s, p)$ and $b(n, s, p)$ are defined in the Appendix, where certain particular values of them are also given. The expressions for $B_{\mu s}^{M n}$ and $D_{\mu s}^{M n}$, Eqs. (30) and (32), differ by a minus sign from Cruzan's values [6], as discussed in the Appendix.

With $O_{1} O_{2}$ along the $z$ axis one has $\theta_{0}=\pi, \theta_{0}^{\prime}=0$. Therefore, $P_{p}^{M-\mu}(\cos \pi)=P_{p}^{M-\mu}(\cos$ $0)=0$ for $\mu \neq M$ and $P_{p}(\cos \pi)=(-1)^{p}=(-1)^{n+s}, P_{p}(\cos 0)=1$. As a result the expressions (29)-(32) simplify to

$$
\begin{aligned}
A_{M s}^{M n}=(-1)^{n+s} C_{M s}^{M n}=(-1)^{M+n+s} \sum_{p=|n-s|}^{n+s} a(M, n|-M, s| p) a(n, s, p) j_{p}(k d), \\
B_{M s}^{M n}=(-1)^{n+s+1} D_{M s}^{M n}=(-1)^{M+n+s+1} \\
\cdot \sum_{p=|n-s|}^{n+s} a(M, n|-M, s| p+1, p) b(n, s, p+1) j_{p+1}(k d),
\end{aligned}
$$

while in (25)-(28) $\mu$ takes only the value $\mu=M$, forcing $s$ to start from $s \geq|M|$. Substituting in (23), (24), interchanging the summations over $s$ and $n$ and using the relations

$$
\hat{r} \times \bar{m}_{M s}=[s(s+1)]^{1 / 2} z_{s}(k r) \bar{B}_{M s}, \hat{r} \times \bar{n}_{M s}=-[s(s+1)]^{1 / 2} \frac{z_{s}^{d}(k r)}{k r} \bar{C}_{M s},
$$


one finally obtains:

$$
\begin{aligned}
x_{1} & \sum_{s=\mid M}^{\infty}[s(s+1)]^{1 / 2}\left[-F_{M s} \frac{h_{s}\left(x_{1}\right) j_{s}^{d}\left(x_{1}\right)}{x_{1} j_{s}\left(x_{1}\right)} \bar{C}_{M s}\left(\theta_{1}, \phi_{1}\right)+A_{M s} \frac{h_{s}^{d}\left(x_{1}\right)}{j_{s}^{d}\left(x_{1}\right)} j_{s}\left(x_{1}\right) \bar{B}_{M s}\left(\theta_{1}, \phi_{1}\right)\right] \\
& +x_{2} \sum_{s=|M|}^{\infty}[s(s+1)]^{1 / 2} \sum_{n=|M|}^{\infty}\left\{H _ { M n } \frac { h _ { n } ( x _ { 2 } ) } { j _ { n } ( x _ { 2 } ) } \left[-A_{M s}^{M n} \frac{j_{s}^{d}\left(x_{1}\right)}{x_{1}} \bar{C}_{M s}\left(\theta_{1}, \phi_{1}\right)\right.\right. \\
& \left.+B_{M s}^{M n} j_{s}\left(x_{1}\right) \bar{B}_{M s}\left(\theta_{1}, \phi_{1}\right)\right]+G_{M n} \frac{h_{n}^{d}\left(x_{2}\right)}{j_{n}^{d}\left(x_{2}\right)}\left[A_{M s}^{M n} j_{s}\left(x_{1}\right) \bar{B}_{M s}\left(\theta_{1}, \phi_{1}\right)\right. \\
& \left.\left.-B_{M s}^{M n} \frac{j_{s}^{d}\left(x_{1}\right)}{x_{1}} \bar{C}_{M s}\left(\theta_{1}, \phi_{1}\right)\right]\right\}=0, \\
x_{1} & \sum_{s=|M|}^{\infty}[s(s+1)]^{1 / 2} \sum_{n=|M|}^{\infty}\left\{F_{M n}\left[-C_{M s}^{M n} \frac{h_{s}^{d}\left(x_{2}\right)}{x_{2}} \bar{C}_{M s}\left(\theta_{2}, \phi_{2}\right)+D_{M s}^{M n} h_{s}\left(x_{2}\right) \bar{B}_{M s}\left(\theta_{2}, \phi_{2}\right)\right]\right. \\
& \left.+A_{M n}\left[C_{M n}^{M n} h_{s}\left(x_{2}\right) \bar{B}_{M s}\left(\theta_{2}, \phi_{2}\right)-D_{M s}^{M n} \frac{h_{s}^{d}\left(x_{2}\right)}{x_{2}} \bar{C}_{M s}\left(\theta_{2}, \phi_{2}\right)\right]\right\}+\sum_{s=|M|}^{\infty}[s(s+1)]^{1 / 2} \\
& {\left[-H_{M s} h_{s}^{d}\left(x_{2}\right) \bar{C}_{M s}\left(\theta_{2}, \phi_{2}\right)+G_{M s} x_{2} h_{s}\left(x_{2}\right) \bar{B}_{M s}\left(\theta_{2}, \phi_{2}\right)\right]=0 . }
\end{aligned}
$$

Invoking the orthogonal properties of the $\bar{B}_{M s}$ and $\bar{C}_{M s}$ vectors over the spherical surfaces $S_{1}$ and $S_{2}$, one finally gets four sets of homogeneous linear equations for the expansion coefficients $A_{M s}, F_{M s}, G_{M s}, H_{M s}$ of $\overline{j_{1}}\left(p_{1}\right), \overline{j_{2}}\left(p_{2}\right)$. The last two, originating from (36), express $G_{M s}, H_{M s}$ in terms of $A_{M s}, F_{M s}$. When substituted back into the first two equations, originating from (35), they produce two sets of homogeneous linear equations for the determination of $A_{M s}, F_{M s}$. The final formulas are:

$$
\begin{gathered}
G_{M s}=-\frac{x_{1}}{x_{2}} \sum_{n=|M|}^{\infty}\left(F_{M n} D_{M s}^{M n}+A_{M n} C_{M s}^{M n}\right), H_{M s}=-\frac{x_{1}}{x_{2}} \sum_{n=|M|}^{\infty}\left(F_{M n} C_{M s}^{M n}+A_{M n} D_{M s}^{M n}\right), \\
A_{M s} \frac{h_{s}^{d}\left(x_{1}\right)}{j_{s}^{d}\left(x_{1}\right)}-\sum_{\nu=|M|}^{\infty} A_{M \nu} \sum_{n=|M|}^{\infty}\left[\frac{h_{n}\left(x_{2}\right)}{j_{n}\left(x_{2}\right)} B_{M s}^{M n} D_{M n}^{M \nu}+\frac{h_{n}^{d}\left(x_{2}\right)}{j_{n}^{d}\left(x_{2}\right)} A_{M s}^{M n} C_{M n}^{M \nu}\right]-\sum_{\nu=|M|}^{\infty} F_{M \nu} \\
\cdot \sum_{n=|M|}^{\infty}\left[\frac{h_{n}\left(x_{2}\right)}{j_{n}\left(x_{2}\right)} B_{M s}^{M n} C_{M n}^{M \nu}+\frac{h_{n}^{d}\left(x_{2}\right)}{j_{n}^{d}\left(x_{2}\right)} A_{M s}^{M n} D_{M n}^{M \nu}\right]=0, \\
F_{M s} \frac{h_{s}\left(x_{1}\right)}{j_{s}\left(x_{1}\right)}-\sum_{\nu=|M|}^{\infty} A_{M \nu} \sum_{n=|M|}^{\infty}\left[\frac{h_{n}\left(x_{2}\right)}{j_{n}\left(x_{2}\right)} A_{M s}^{M n} D_{M n}^{M \nu}+\frac{h_{n}^{d}\left(x_{2}\right)}{j_{n}^{d}\left(x_{2}\right)} B_{M s}^{M n} C_{M n}^{M \nu}\right]-\sum_{\nu=|M|}^{\infty} F_{M \nu} \\
\cdot \sum_{n=|M|}^{\infty}\left[\frac{h_{n}\left(x_{2}\right)}{j_{n}\left(x_{2}\right)} A_{M s}^{M n} C_{M n}^{M \nu}+\frac{h_{n}^{d}\left(x_{2}\right)}{j_{n}^{d}\left(x_{2}\right)} B_{M s}^{M n} D_{M n}^{M \nu}\right]=0 .
\end{gathered}
$$

Setting the determinant of the coefficients equal to 0 provides the equation from which the resonant values $k_{n s}=\omega_{n s} / c$ are determined. For general values of $k d$ one can procede from here by numerical methods only. The complications and uncertainties of such an approach are discussed fully in [1].

For small values of $k d$, however, an analytical solution is possible. In particular, for $k d$ $=0$ it is obvious from (33), (34) and the fact that $j_{n}(0)=0, n \neq 0, j_{0}(0)=1$ that $B_{M s}^{M n}=D_{M s}^{M n}$ $=0$ and $A_{M s}^{M n}=C_{M s}^{M n}=\delta_{n s}$, where $\delta_{n s}=0$ for $n \neq s$ and $\delta_{s s}=1$. The last result follows from (25)-(28) if it is noticed that for $d=0$ there is no translation and that the eigenvectors $\bar{m}, \bar{n}$ 
form a complete and orthogonal set. It follows from (37)-(39) that

$$
\begin{gathered}
G_{M s}=-\left(x_{1} / x_{2}\right) A_{M s}, H_{M s}=-\left(x_{1} / x_{2}\right) F_{M s}(s=|M|,|M|+1, \cdots), \\
\frac{h_{s}^{d}\left(x_{1}\right)}{j_{s}^{d}\left(x_{1}\right)}-\frac{h_{s}^{d}\left(x_{2}\right)}{j_{s}^{d}\left(x_{2}\right)}=0 \text { (electric modes); } \frac{h_{s}\left(x_{1}\right)}{j_{s}\left(x_{1}\right)}-\frac{h_{s}\left(x_{2}\right)}{j_{s}\left(x_{2}\right)}=0 \text { (magnetic modes). }
\end{gathered}
$$

The resonant frequencies $\omega_{s n}(0)$ are found from the solution of Eqs. (41) $(s=|M|,|M|+$ $1, \cdots ; n=1,2, \cdots)$ and are independent of $M$ (apart from the restriction $s \geq|M|$ ). Moreover, there is no coupling between electric and magnetic modes. All these results are well known from separation of variables.

For $k d \neq 0$, but small, it is observed from (33), (34) and the relations

$j_{n}(k d) \simeq \frac{2^{n} n !}{(2 n+1) !}(k d)^{n}\left[1+O\left(k^{2} d^{2}\right)\right] \quad$ for $n \neq 0, \quad j_{0}(k d) \simeq 1-\frac{(k d)^{2}}{6}+O\left(k^{4} d^{4}\right)$

that for $n \neq s$ and $n=s$

$$
A_{M s}^{M n}, C_{M s}^{M n} \sim(k d)^{|n-s|}\left[1+O\left(k^{2} d^{2}\right)\right] ; B_{M s}^{M n}, D_{M s}^{M n} \sim(k d)^{|n-s|+1}\left[1+O\left(k^{2} d^{2}\right)\right] .
$$

These results, as in [1], simplify Eqs. (37)-(39) a great deal, but not to the extent achieved in [1]. The reason is that for $M \neq 0$ the coefficients $A_{M s}$ remain coupled to the $F_{M s}$ (an $\bar{m}$ or $\bar{n}$ mode is expanded under translation into both $\bar{m}$ and $\bar{n}$ modes, unlike the scalar case, Eqs. (20) or (21), (22) in [1]). Omitting details, it is found that in the determinant $D\left(a_{n s}\right)$ of the coefficients of $A_{M s}, F_{M s}$ terms of order $k d$ that must be retained are found not only along the diagonal $\left(a_{s s}\right)$ and the ones next to it $\left(a_{s, s+1} ; a_{s+1, s}\right)$, as in [1], but, in addition, along the next two diagonals $\left(a_{s, s+2} ; a_{s+2, s}\right)$. The evaluation of $D\left(a_{n s}\right)$ in such a case can still be carried out, as will be shown in a forthcoming publication on the exterior (scattering) problem. However, here these difficulties can be avoided if one restricts the examination to the case $M=0$. It is to be remembered here that the axial symmetry of the configuration makes this the most important case, since azimuthal dependence $(M \neq 0)$ may be introduced only by an initial non-axisymmetric impressed field that generates the oscillations inside the cavity, not by the configuration. For $M=0$ the $\bar{m}$ and $\bar{n}$ modes become completely decoupled. Indeed, from (34) and (A.5) of the Appendix (and for unrestricted values of $k d$ ) one has:

$$
a(0, n|0, s| p+1, p)=0 ; \quad B_{0 s}^{o n}=D_{0 s}^{o n}=0 .
$$

These values imply that in (25)-(28) the $\bar{m} / \bar{n}$ modes are expanded under translation into $\bar{m} / \bar{n}$ modes only, while (37)-(39) simplify to:

$$
\begin{gathered}
G_{0 s}=-\frac{x_{1}}{x_{2}} \sum_{n=1}^{\infty} C_{0 s}^{0 n} A_{0 n} ; \quad H_{0 s}=-\frac{x_{1}}{x_{2}} \sum_{n=1}^{\infty} C_{0 s}^{0 n} F_{0 n}(s=1,2, \cdots), \\
A_{0 s} \frac{h_{s}^{d}\left(x_{1}\right)}{j_{s}^{d}\left(x_{2}\right)}-\sum_{\nu=1}^{\infty} A_{0 \nu} \sum_{n=1}^{\infty} \frac{h_{n}^{d}\left(x_{2}\right)}{j_{n}^{d}\left(x_{2}\right)} A_{0 s}^{0 n} C_{0 n}^{0 \nu}=0, \\
F_{0 s} \frac{h_{s}\left(x_{1}\right)}{j_{s}\left(x_{1}\right)}-\sum_{\nu=1}^{\infty} F_{0 \nu} \sum_{n=1}^{\infty} \frac{h_{n}\left(x_{2}\right)}{j_{n}\left(x_{2}\right)} A_{0 s}^{0 n} C_{0 n}^{0 \nu}=0 .
\end{gathered}
$$

In these equations the indices $s, n$ start from $n, s=1$ (not 0 ), since $\bar{m}_{00}=\bar{n}_{00} \equiv 0$, as seen from (4), (5). Both (46) and (47) are similar to (29) or (67) in [1] and can be put into the 
forms

$$
\begin{gathered}
\sum_{\nu=1}^{\infty} a_{s \nu} A_{0 \nu}=0, \quad \sum_{\nu=1}^{\infty} f_{s \nu} F_{0 \nu}=0 ; \quad \nu, s=1,2, \cdots, \\
a_{s s}=\frac{h_{s}^{d}\left(x_{1}\right)}{j_{s}^{d}\left(x_{1}\right)}-\sum_{n=1}^{\infty} \frac{h_{n}^{d}\left(x_{2}\right)}{j_{n}^{d}\left(x_{2}\right)} A_{0 s}^{0 n} C_{0 n}^{0 s}, \quad a_{s \nu}=-\sum_{n=1}^{\infty} \frac{h_{n}^{d}\left(x_{2}\right)}{j_{n}^{d}\left(x_{2}\right)} A_{0 s}^{0 n} C_{0 n}^{0 \nu}(s \neq \nu), \\
f_{s s}=\frac{h_{s}\left(x_{1}\right)}{j_{s}\left(x_{1}\right)}-\sum_{n=1}^{\infty} \frac{h_{n}\left(x_{2}\right)}{j_{n}\left(x_{2}\right)} A_{0 s}^{0 n} C_{0 n}^{0 s}, \quad f_{s \nu}=-\sum_{n=1}^{\infty} \frac{h_{n}\left(x_{2}\right)}{j_{n}\left(x_{2}\right)} A_{0 s}^{0 n} C_{0 n}^{0 \nu}(s \neq \nu),
\end{gathered}
$$

similar to (30)-(32) in [1]. From here on it is obviously possible to treat only the second case for the coefficients $f_{s \nu}$ (magnetic modes), and obtain results for the $a_{s v}$ (electric modes) by mere substitution of $z_{n}\left(x_{1}\right), z_{n}\left(x_{2}\right)$ by $z_{n}^{d}\left(x_{1}\right), z_{n}^{d}\left(x_{2}\right)$, respectively, $z_{n}$ being either $j_{n}$ or $h_{n}$.

For small $k d$, reference to (33), (42), (43) shows that

$$
A_{0 s}^{0 n} C_{0 n}^{0 \nu} \sim(k d)^{|s-n|+|\nu-n|}\left[1+O\left(k^{2} d^{2}\right)\right],
$$

implying that

$$
f_{s s}=C_{s s}+C_{s s}^{\prime \prime}(k d)^{2}+O\left(k^{4} d^{4}\right) ; \quad f_{s \nu}=C_{s \nu}^{\prime}(k d)^{|s-\nu|}\left[1+O\left(k^{2} d^{2}\right)\right], \quad(\nu \neq s),
$$

in which the C's are independent of $k d$. From this point on the procedure follows steps identical to [1], leading to an explicit evaluation of the determinant $D\left(f_{s v}\right)$ to order $(k d)^{2}$ (Eq. (41) in [1]) and to a closed-form expression for the coefficients $g_{v s}$ in the relation $\omega_{\nu s}(d)=\omega_{\nu s}(0) \cdot\left[1+g_{\nu s}(k d)^{2}\right]$ for the resonant frequencies. The latter (Eq. (55) in [1]) is repeated here for convenience:

$$
g_{\nu s}=\left[x_{1} \frac{d C_{\nu \nu}\left(x_{1}\right)}{d x_{1}}\right]^{-1}\left[\frac{C_{\nu+1, \nu}^{\prime} C_{\nu, \nu+1}^{\prime}}{C_{\nu+1, \nu+1}}+\frac{C_{\nu, \nu-1}^{\prime} C_{\nu-1, \nu}^{\prime}}{C_{\nu-1, \nu-1}}-C_{\nu \nu}^{\prime \prime}\right] \quad(\nu, s=1,2, \cdots),
$$

where, in differentiating $d C_{v v}\left(x_{1}, x_{2}\right) / d x_{1}$, one writes $x_{2}=r x_{1}$ with $r=x_{2} / x_{1}=R_{2} / R_{1}=$ constant.

There remain the explicit expressions for the constants $C$. They are obtained from (33), (42), (51) and (52):

$$
\begin{aligned}
f_{n n}= & C_{n n}+C_{n n}^{\prime \prime}(k d)^{2}+O\left(k^{4} d^{4}\right)=\frac{h_{n}\left(x_{1}\right)}{j_{n}\left(x_{1}\right)}-\frac{h_{n}\left(x_{2}\right)}{j_{n}\left(x_{2}\right)} A_{0 n}^{0 n} C_{0 n}^{0 n}-\frac{h_{n-1}\left(x_{2}\right)}{j_{n-1}\left(x_{2}\right)} A_{0 n}^{0, n-1} C_{0, n-1}^{0 n} \\
& -\frac{h_{n+1}\left(x_{2}\right)}{j_{n+1}\left(x_{2}\right)} A_{0 n}^{0, n+1} C_{0, n+1}^{0 n}+O\left(k^{4} d^{4}\right) \\
f_{n, n+1} & =-\frac{h_{n}\left(x_{2}\right)}{j_{n}\left(x_{2}\right)} A_{0 n}^{0 n} C_{0 n}^{0, n+1}-\frac{h_{n+1}\left(x_{2}\right)}{j_{n+1}\left(x_{2}\right)} A_{0 n}^{0, n+1} C_{0, n+1}^{0, n+1}+O\left(k^{3} d^{3}\right) \\
& =C_{n, n+1}^{\prime}(k d)\left[1+O\left(k^{2} d^{2}\right)\right], \\
f_{n+1, n} & =-\frac{h_{n}\left(x_{2}\right)}{j_{n}\left(x_{2}\right)} A_{0, n+1}^{0 n} C_{0 n}^{0 n}-\frac{h_{n+1}\left(x_{2}\right)}{j_{n+1}\left(x_{2}\right)} A_{0, n+1}^{0, n+1} C_{0, n+1}^{0 n}+O\left(k^{3} d^{3}\right) \\
& =C_{n+1, n}^{\prime}(k d)\left[1+O\left(k^{2} d^{2}\right)\right] .
\end{aligned}
$$

Using from the Appendix formulas for $a(0, n|0, s| p) a(n, s, p)$ one gets:

$A_{0 n}^{o n} C_{0 n}^{0 n}=\left[a(0, n|0, n| 0) a(n, n, 0)\left(1-k^{2} d^{2} / 6\right)\right.$ 


$$
\begin{aligned}
+ & \left.a(0, n|0, n| 1) a(n, n, 2)(k d)^{2} / 15+O\left(k^{4} d^{4}\right)\right]^{2} \\
=1- & \frac{(k d)^{2}}{3}\left[1+\frac{2\left(n^{2}+n-3\right)}{(2 n+3)(2 n-1)}\right]+O\left(k^{4} d^{4}\right) \\
A_{0, n}^{0, n-1} C_{0, n-1}^{0 n}= & {\left[-a(0, n-1|0, n| 1) a(n-1, n, 1)(k d / 3)+O\left(k^{3} d^{3}\right)\right][a(0, n|0, n-1| 1)} \\
& \left.\cdot a(n, n-1,1)(k d / 3)+O\left(k^{3} d^{3}\right)\right]=\frac{n^{2}-1}{4 n^{2}-1}(k d)^{2}+O\left(k^{4} d^{4}\right), \\
A_{0, n+1}^{0, n+1} C_{0, n+1}^{0 n}=[ & {\left[-a(0, n+1|0, n| 1) a(n+1, n, 1)(k d / 3)+O\left(k^{3} d^{3}\right)\right][a(0, n|0, n+1| 1)} \\
& \left.\cdot a(n, n+1,1)(k d / 3)+O\left(k^{3} d^{3}\right)\right]=\frac{n(n+2)}{(2 n+1)(2 n+3)}(k d)^{2}+O\left(k^{4} d^{4}\right) .
\end{aligned}
$$

Therefore:

$$
\begin{aligned}
C_{n n}= & \frac{h_{n}\left(x_{1}\right)}{j_{n}\left(x_{1}\right)}-\frac{h_{n}\left(x_{2}\right)}{j_{n}\left(x_{2}\right)}=i\left[\frac{n_{n}\left(x_{1}\right)}{j_{n}\left(x_{1}\right)}-\frac{n_{n}\left(x_{2}\right)}{j_{n}\left(x_{2}\right)}\right]=f_{n n}(0), \\
C_{n n}^{\prime \prime}= & \frac{(2 n+3)(2 n-1)+2 n^{2}+2 n-6}{3(2 n+3)(2 n-1)} \frac{h_{n}\left(x_{2}\right)}{j_{n}\left(x_{2}\right)}-\frac{n^{2}-1}{4 n^{2}-1} \frac{h_{n-1}\left(x_{2}\right)}{j_{n-1}\left(x_{2}\right)} \\
& -\frac{n(n+2)}{(2 n+1)(2 n+3)} \frac{h_{n+1}\left(x_{2}\right)}{j_{n+1}\left(x_{2}\right)} \\
= & i\left\{\left[\frac{n^{2}-1}{4 n^{2}-1}+\frac{n(n+2)}{(2 n+1)(2 n+3)}\right] \frac{n_{n}\left(x_{2}\right)}{j_{n}\left(x_{2}\right)}-\frac{n^{2}-1}{4 n^{2}-1} \frac{n_{n-1}\left(x_{2}\right)}{j_{n-1}\left(x_{2}\right)}\right. \\
& \left.-\frac{n(n+2)}{(2 n+1)(2 n+3)} \frac{n_{n+1}\left(x_{2}\right)}{j_{n+1}\left(x_{2}\right)}\right\} .
\end{aligned}
$$

Also, following similar steps:

$$
\begin{aligned}
& C_{n, n+1}^{\prime}=\frac{n+2}{2 n+3}\left[\frac{h_{n+1}\left(x_{2}\right)}{j_{n+1}\left(x_{2}\right)}-\frac{h_{n}\left(x_{2}\right)}{j_{n}\left(x_{2}\right)}\right]=i \frac{n+2}{2 n+3}\left[\frac{n_{n+1}\left(x_{2}\right)}{j_{n+1}\left(x_{2}\right)}-\frac{n_{n}\left(x_{2}\right)}{j_{n}\left(x_{2}\right)}\right], \\
& C_{n+1, n}^{\prime}=\frac{n}{2 n+1}\left[\frac{h_{n+1}\left(x_{2}\right)}{j_{n+1}\left(x_{2}\right)}-\frac{h_{n}\left(x_{2}\right)}{j_{n}\left(x_{2}\right)}\right]=i \frac{n}{2 n+1}\left[\frac{n_{n+1}\left(x_{2}\right)}{j_{n+1}\left(x_{2}\right)}-\frac{n_{n}\left(x_{2}\right)}{j_{n}\left(x_{2}\right)}\right] .
\end{aligned}
$$

As in [1], one obtains the remarkable result that all the C's turn out to be imaginary. This means that all elements $f_{\nu s}$ of the determinant $D\left(f_{\nu s}\right)$, at least to order $(k d)^{2}$, are imaginary, and therefore that the $\omega_{n s}(d)$ are real, as predicted by theory for all cavities. This is a very convincing check on all relations and results obtained in this paper.

For the electric modes one simply replaces $j_{n}(x)$ and $n_{n}(x)$ in (60)-(63) by $j_{n}^{d}(x)=$ $\left[x j_{n}(x)\right]^{\prime}$ and $n_{n}^{d}(x)=\left[x n_{n}(x)\right]^{\prime}$. Comparing with the results of [1] for $M=0$ one observes that the $\omega_{n s}(d)$ for the magnetic modes are no longer the same with those of the Dirichlet scalar case, as happens to be true for concentric spheres $(d=0)$.

Numerical results. The roots $\left(x_{1}\right)_{\nu s}(\nu=1,2, \cdots ; s=1,2, \cdots)$ of $(41)$ were obtained by a modified Newton-Raphson method, called the Regula-Falsi method. In the case of the magnetic modes these roots are the same as those of the Dirichlet scalar problem and have been checked against tabulated values, as explained in [1]. For $M=0$ the roots $\left(x_{1}\right)_{\nu s}$ 
for the magnetic modes and the corresponding $g_{v s}$ from (53) are given in Tables I-IV for four values of the ratio $r=x_{2} / x_{1}=R_{2} / R_{1}$, namely, $r=1.2-1.35-1.5-2.0$. Similar values for the electric modes and for $M=0$ are found in Tables V-VIII.

One general observation is the fact that the $g_{\nu s}$ are almost independent of the order $s$ of the resonant frequency; in other words, the percentage change of $\omega_{\nu s}(d)$ from $\omega_{\nu s}(0)$ is almost the same for all $s$ (same $\nu$ ), particularly for $s \geq 2$. As far as the complications of a direct numerical evaluation of the roots of the equation $D\left(f_{v s}\right)=0$ are concerned, the reader is referred to the detailed discussion of this aspect in [1].

Reference to [1] should also be made concerning the possible generalization of the present approach to exterior problems (scattering by an eccentrically coated sphere is at present under investigation), as well as to other geometrical configurations.

Recently, cylindrical geometries were considered, in particular eccentric waveguides and eccentrically coated waveguides. Our results were found in excellent agreement with those of [9], in which the cutoff frequencies of the lower TM and TE modes were obtained numerically and experimentally for eccentric, perfectly conducting waveguides. For small $k d$ agreement to four decimals was obtained. Even for the largest $k d$ considered in [9] the agreement extended to the first two decimals, an indication that the restriction $k d<<1$ of the present method is not really as severe as it may appear. This is further corroborated by the fact that the $g_{v s}$ get smaller quickly with increasing ratio $r$, as seen from the tables. Besides, when $r$ is small and both $g_{v s}$ and $x_{1}$ become relatively large, $k d$ gets necessarily small due to the physical restriction $d \leq R_{2}-R_{1}$ or $k d \leq(r-1) x_{1}$. This application to waveguides will be the subject of a forthcoming paper.

Appendix. The symbols $a(M, n|-\mu, s| p), a(n, s, p)$ etc. appearing in (29)-(32) are defined as follows [6]:

$$
\begin{aligned}
& a(m, n|\mu, s| p)=(-1)^{m+\mu}(2 p+1)\left[\frac{(n+m) !(s+\mu) !(p-m-\mu) !}{(n-m) !(s-\mu) !(p+m+\mu) !}\right]^{1 / 2} \\
& \cdot\left[\begin{array}{lll}
n & s & p \\
0 & 0 & 0
\end{array}\right]\left[\begin{array}{ccc}
n & s & p \\
m & \mu & -m-\mu
\end{array}\right] \\
& a(m, n|\mu, s| p+1, q)=(-1)^{m+\mu}(2 p+3)\left[\frac{(n+m) !(s+\mu) !(p+1-m-\mu) !}{(n-m) !(s-\mu) !(p+1+m+\mu) !}\right]^{1 / 2} \\
& \cdot\left[\begin{array}{lll}
n & s & q \\
0 & 0 & 0
\end{array}\right]\left[\begin{array}{ccc}
n & s & p+1 \\
m & \mu & -m-\mu
\end{array}\right], \\
& a(n, s, p)=i^{p+s-n}[2 s(s+1)(2 s+1)+(s+1)(n-s+p+1)(n+s-p) \\
&-s(s-n+p+1)(n+s+p+2)] /[2 s(s+1)] \\
& b(n, s, q)=i^{s+q-n}\left[q^{2}-(s-n)^{2}\right]^{1 / 2}\left[(s+n+1)^{2}-q^{2}\right]^{1 / 2}(2 s+1) /[2 s(s+1)], \quad \text { (A }
\end{aligned}
$$

where

$$
\left[\begin{array}{ccc}
j_{1} & j_{2} & j_{3} \\
m_{1} & m_{2} & m_{3}
\end{array}\right]
$$

is the Wigner $3-j$ symbol. Some useful properties of the latter are: the symbol is 0 unless $m_{1}+m_{2}+m_{3}=0$, unless the triangular condition $\left|j_{1}-j_{2}\right| \leq j_{3} \leq j_{1}+j_{2}$ is satisfied 
Magnetic modes $(\mathrm{M}=0)$

TABLE I. $r=x_{2} / x_{1}=1.2$.

\begin{tabular}{|c|c|c|c|c|c|c|c|}
\hline & & $s=1$ & $s=2$ & $s=3$ & $s=4$ & $s=5$ & $s=6$ \\
\hline \multirow{5}{*}{$\left(\mathrm{x}_{1}\right)_{\text {vs }}$} & $\nu=1$ & 15.76063 & 31.44237 & 47.14150 & 62.84508 & 78.55033 & 94.2565 \\
\hline & 2 & 15.86550 & 31.49526 & 47.17682 & 62.87154 & 78.57154 & 94.27419 \\
\hline & 3 & 16.02151 & 31.57444 & 47.22976 & 62.91128 & 78.60335 & 94.3007 \\
\hline & 4 & 16.22717 & 31.67971 & 47.30025 & 62.96423 & 78.64574 & 94.33604 \\
\hline & 5 & 16.48061 & 31.81082 & 47.38822 & 63.03036 & 78.69869 & 94.38019 \\
\hline \multirow{5}{*}{$\mathrm{g}_{\mathrm{us}}$} & $\nu=1$ & -2.94460 & -2.98393 & -2.98868 & -2.98745 & -2.98846 & -2.97993 \\
\hline & 2 & .7531856 & .7275187 & .7258693 & .7285967 & .7340752 & .7403467 \\
\hline & 3 & .5301020 & .5099873 & .5074118 & .5089340 & .5115610 & .5158321 \\
\hline & 4 & .3564039 & .3393728 & .3365035 & .3368490 & .3381674 & .3404283 \\
\hline & 5 & .2524817 & .2378851 & .2350669 & .2348713 & .2360829 & .2375913 \\
\hline
\end{tabular}

TABLE II. $\mathrm{r}=\mathrm{x}_{2} / \mathrm{x}_{1}=1.35$.

\begin{tabular}{|c|c|c|c|c|c|c|c|}
\hline & & $s=1$ & $s=2$ & $s=3$ & $s=4$ & $\mathrm{~s}=5$ & $s=6$ \\
\hline \multirow{6}{*}{$\left(\mathrm{x}_{1}\right)_{v \mathrm{~s}}$} & $\nu=1$ & 9.057011 & 17.99305 & 26.95539 & 35.92452 & 44.89639 & 53.86962 \\
\hline & 2 & 9.216926 & 18.07494 & 27.01022 & 35.96571 & 44.92936 & 53.89711 \\
\hline & 3 & 9.451597 & 18.19702 & 27.09224 & 36.02739 & 44.97876 & 53.93831 \\
\hline & 4 & 9.755591 & 18.35861 & 27.20123 & 36.10947 & 45.04456 & 53.99319 \\
\hline & 5 & 10.12253 & 18.55865 & 27.33688 & 36.21183 & 45.12667 & 54.06172 \\
\hline & & $s=1$ & $s=2$ & $\mathrm{~s}=3$ & $s=4$ & $\mathrm{~s}=5$ & $s=6$ \\
\hline \multirow{5}{*}{$g_{\nu 8}$} & $\nu=1$ & -1.04854 & -1.08817 & -1.09551 & -1.09844 & -1.09953 & -1.10042 \\
\hline & 2 & .2976899 & .2714140 & .2665779 & .2650669 & .2642967 & .2632253 \\
\hline & 3 & .2090797 & .1917095 & .1874811 & .1858838 & .1851030 & .1847804 \\
\hline & 4 & .1406013 & .1285457 & .1249803 & .1235807 & .1229980 & .1225309 \\
\hline & 5 & .0987714 & .0909096 & .0878274 & .0865854 & .0860460 & .0856145 \\
\hline
\end{tabular}

TABLE III. $\mathrm{r}=\mathrm{x}_{2} / \mathrm{x}_{1}=1.5$.

\begin{tabular}{|c|c|c|c|c|c|c|c|}
\hline & & $s=1$ & $s=2$ & $\mathrm{~s}=3$ & $s=4$ & $\mathrm{~s}=5$ & $s=6$ \\
\hline \multirow{5}{*}{$\left(\mathrm{x}_{1}\right)_{\nu \mathrm{s}}$} & $\nu=1$ & 6.38580 & 12.61895 & 18.88476 & 25.15918 & 31.43708 & 37.71673 \\
\hline & 2 & 6.58613 & 12.72351 & 18.95504 & 25.21203 & 31.47942 & 37.75204 \\
\hline & 3 & 6.87535 & 12.87882 & 19.05999 & 25.29113 & 31.54283 & 37.80495 \\
\hline & 4 & 7.24246 & 13.08311 & 19.19908 & 25.39622 & 31.62720 & 37.87539 \\
\hline & 5 & 7.67580 & 13.33421 & 19.37160 & 25.52701 & 31.73236 & 37.96326 \\
\hline \multirow{5}{*}{$\mathrm{g}_{v s}$} & $\nu=1$ & -.548217 & -.585778 & -.593381 & -.596055 & -.597151 & -.597566 \\
\hline & 2 & .1751128 & .1529077 & .1477128 & .1460435 & .1453082 & .1451701 \\
\hline & 3 & .1207802 & .1077643 & .1038657 & .1023786 & .1018547 & .1015902 \\
\hline & 4 & .0795457 & .0726922 & .0695374 & .0682722 & .0676965 & .0674194 \\
\hline & 5 & .0541408 & .0516278 & .0491016 & .0479806 & .0474355 & .0471801 \\
\hline
\end{tabular}


TABLE IV. $r=x_{2} / x_{1}=2.0$.

\begin{tabular}{|c|c|c|c|c|c|c|c|}
\hline & & $s=1$ & $s=2$ & $\mathrm{~s}=3$ & $s=4$ & $s=5$ & $s=6$ \\
\hline \multirow{5}{*}{$\left(\mathrm{X}_{1}\right)_{v \mathrm{~s}}$} & $\nu=1$ & 3.28600 & 6.36067 & 9.47718 & 12.60587 & 15.73963 & 18.87597 \\
\hline & 2 & 3.55578 & 6.51306 & 9.58126 & 12.68459 & 15.80286 & 18.92877 \\
\hline & 3 & 3.92252 & 6.73556 & 9.73553 & 12.80189 & 15.89729 & 19.00774 \\
\hline & 4 & 4.35839 & 7.02183 & 9.93792 & 12.95687 & 16.02247 & 19.11260 \\
\hline & 5 & 4.84099 & 7.36467 & 10.18591 & 13.14838 & 16.17778 & 19.24300 \\
\hline \multirow{5}{*}{$\mathrm{g}_{\nu \mathrm{s}}$} & $\nu=1$ & -.154770 & -.185607 & -.193237 & -.196091 & -.197428 & -.198144 \\
\hline & 2 & .0701890 & .0573415 & .0524788 & .0505131 & .0495400 & .0490610 \\
\hline & 3 & .0429004 & .0402039 & .0371142 & .0356451 & .0348920 & .0344651 \\
\hline & 4 & .0247884 & .0270546 & .0252153 & .0240656 & .0234315 & .0230618 \\
\hline & 5 & .0144915 & .0188940 & .0180254 & .0171499 & .0166155 & .0162764 \\
\hline
\end{tabular}

Electric Modes $(\mathrm{M}=0)$

TABIE V. $\mathrm{r}=\mathrm{x}_{2} / \mathrm{x}_{1}=1.2$.

\begin{tabular}{|c|c|c|c|c|c|c|c|}
\hline & & $s=1$ & $\mathrm{~s}=2$ & $s=3$ & $s=4$ & $s=5$ & $s=6$ \\
\hline \multirow{6}{*}{$\left(\mathrm{x}_{1}\right)_{\nu \mathrm{s}}$} & $\nu=1$ & 15.76117 & 31.44230 & 47.14152 & 62.84504 & 78.55033 & 94.25651 \\
\hline & 2 & 15.86712 & 31.49547 & 47.17688 & 62.87157 & 78.57155 & 94.27419 \\
\hline & 3 & 16.02478 & 31.57485 & 47.22988 & 62.91133 & 78.60337 & 94.30071 \\
\hline & 4 & 16.23269 & 31.68040 & 47.30045 & 62.96431 & 78.64578 & 94.33606 \\
\hline & 5 & 16.48902 & 31.81185 & 47.38852 & 63.03048 & 78.69876 & 94.38023 \\
\hline & & $\mathbf{s}=1$ & $\mathrm{~s}=2$ & $\mathrm{~s}=3$ & $s=4$ & $s=5$ & $s=6$ \\
\hline \multirow{5}{*}{$\mathrm{g}_{\nu \mathrm{s}}$} & $\nu=1$ & -2.91148 & -2.97116 & -2.97599 & -2.96962 & -2.95778 & -2.94446 \\
\hline & 2 & .7471076 & .7255597 & .7258737 & .7286365 & .7364067 & .7448191 \\
\hline & 3 & .5260365 & .5085578 & .5066550 & .5082459 & .5113348 & .5153637 \\
\hline & 4 & .3540509 & .3384358 & .3363047 & .3370900 & .3385343 & .3411405 \\
\hline & 5 & .2511180 & .2373111 & .2348577 & .2349251 & .2356447 & .2372174 \\
\hline
\end{tabular}

TABLE VI. $r=x_{2} / x_{1}=1.35$.

\begin{tabular}{|c|c|c|c|c|c|c|c|}
\hline & & $s=1$ & $s=2$ & $s=3$ & $s=4$ & $s=5$ & $s=6$ \\
\hline \multirow{5}{*}{$\left(\mathrm{x}_{1}\right)_{\nu \mathrm{s}}$} & $\nu=1$ & 9.059273 & 17.99332 & 26.95547 & 35.92456 & 44.89641 & 53.86963 \\
\hline & 2 & 9.223809 & 18.07577 & 27.01047 & 35.96581 & 44.92941 & 53.89714 \\
\hline & 3 & 9.465746 & 18.19879 & 27.09276 & 36.02761 & 44.97888 & 53.93837 \\
\hline & 4 & 9.779913 & 18.36159 & 27.20211 & 36.10984 & 45.04475 & 53.99330 \\
\hline & 5 & 10.16033 & 18.56318 & 27.33821 & 36.21238 & 45.12695 & 54.06189 \\
\hline \multirow{5}{*}{$g_{v s}$} & $\nu=1$ & -1.01467 & -1.07988 & -1.09213 & -1.09625 & -1.09828 & -1.09950 \\
\hline & 2 & .2935301 & .2707237 & .2663895 & .2646275 & .2638187 & .2637762 \\
\hline & 3 & .2067111 & .1905825 & .1868240 & .1856129 & .1849674 & .1844813 \\
\hline & 4 & .1398599 & .1278972 & .1246793 & .1233498 & .1228392 & .1226116 \\
\hline & 5 & .0989798 & .0905222 & .0876058 & .0864837 & .0858591 & .0855934 \\
\hline
\end{tabular}


TABLE VII. $r=x_{2} / x_{1}=1.5$.

\begin{tabular}{|c|c|c|c|c|c|c|c|}
\hline & & $s=1$ & $s=2$ & $s=3$ & $s=4$ & $s=5$ & $s=6$ \\
\hline \multirow{5}{*}{$\left(\mathrm{x}_{1}\right)_{\mathrm{vg}}$} & $\nu=1$ & 6.39111 & 12.69165 & 18.88497 & 25.15926 & 31.43712 & 37.71676 \\
\hline & 2 & 6.60254 & 12.72563 & 18.95567 & 25.21230 & 31.47956 & 37.75212 \\
\hline & 3 & 6.90955 & 12.88312 & 19.06126 & 25.29166 & 31.54311 & 37.80511 \\
\hline & 4 & 7.30225 & 13.09045 & 19.20122 & 25.39712 & 31.62766 & 37.87565 \\
\hline & 5 & 7.77013 & 13.34553 & 19.37485 & 25.52837 & 31.73305 & 37.96366 \\
\hline \multirow{5}{*}{$\mathrm{gus}_{\mathrm{s}}$} & $\nu=1$ & -.511572 & -.577275 & -.589400 & -.593255 & -.594819 & -.595524 \\
\hline & 2 & .1730606 & .1514145 & 1469667 & .1455730 & 1450011 & .1450366 \\
\hline & 3 & .1203757 & 1068492 & .1033312 & .1020863 & .1015466 & .1013812 \\
\hline & 4 & .0805281 & .0722090 & .0692174 & .0680568 & .0675526 & .0673707 \\
\hline & 5 & .0557589 & .0514180 & .0489064 & .0478255 & .0473364 & .0470939 \\
\hline
\end{tabular}

TABLE VIII. $r=x_{2} / x_{1}=2.0$.

\begin{tabular}{cccccccc}
\hline & & $\mathrm{s}=1$ & $\mathrm{~s}=2$ & $\mathrm{~s}=3$ & $\mathrm{~s}=4$ & $\mathrm{~s}=5$ & $\mathrm{~s}=6$ \\
\hline & $\nu=1$ & 3.30922 & 6.36402 & 9.47820 & 12.60631 & 15.73986 & 18.87610 \\
$\left(\mathrm{x}_{1}\right)_{\text {rs }}$ & 2 & 3.63013 & 6.52362 & 9.58440 & 12.68592 & 15.80353 & 18.92917 \\
& 3 & 4.08033 & 6.75823 & 9.74207 & 12.80460 & 15.89867 & 19.00853 \\
& 4 & 2.95404 & 7.06326 & 9.94941 & 12.96154 & 16.02481 & 19.11394 \\
& 5 & 3.53114 & 7.43401 & 10.2043 & 13.15567 & 16.18139 & 19.24505 \\
\hline & $\nu=1$ & -.109733 & -.175373 & -.188952 & -.193740 & -.195908 & -.196986 \\
& 2 & .0739110 & .0569686 & .0519261 & .0500943 & .0492965 & .0488182 \\
$\mathrm{~g}_{\text {us }}$ & 3 & .0441847 & .0405678 & .0368313 & .0353987 & .0346946 & .0343293 \\
& 4 & -.013230 & .0281633 & .0251838 & .0239423 & .0233305 & .0229799 \\
& 5 & -.009227 & .0205987 & .0182036 & .0171116 & .0165553 & .0162232 \\
\hline
\end{tabular}

cyclically by all indices $j_{1}, j_{2}, j_{3}$ and unless $\left|m_{n}\right| \leq j_{n}$. Moreover,

$$
\left[\begin{array}{ccc}
j_{1} & j_{2} & j_{3} \\
0 & 0 & 0
\end{array}\right]=0
$$

unless $j_{1}+j_{2}+j_{3}=$ even.

It is this last property that makes

$$
a(0, n|0, s| p+1, p)=0,
$$

since it contains, according to (A.2), the factors

$$
\left[\begin{array}{lll}
n & s & p \\
0 & 0 & 0
\end{array}\right]\left[\begin{array}{ccc}
n & s & p+1 \\
0 & 0 & 0
\end{array}\right] .
$$

The symbols $a(m, n|\mu, s| p)$ appear also in the expansion

$$
P_{n}^{m} P_{s}^{\mu}=\sum_{p} a(m, n|\mu, s| p) P_{p}^{m+\mu} \quad(p=n+s, n+s-2, \cdots|n-s|) .
$$

In particular, for $m=\mu=0$ : 


$$
\begin{aligned}
a(0, n|0, s| p)= & (2 p+1) \frac{(n+s-p) !(n+p-s) !(s+p-n) !}{(n+s+p+1) !} \\
& \cdot\left\{\frac{[(n+s+p) / 2] !}{[(n+s-p) / 2] ![(n+p-s) / 2] ![(s+p-n) / 2] !}\right\}^{2},
\end{aligned}
$$

an equation that has been used extensively in the derivation of (57)-(59).

Finally, an independent check of the minus sign correction in Cruzan's formulas for $B_{\mu s}^{m n}, D_{\mu s}^{m n}$ (Eqs. (30) and (32) in this paper) can be provided by evaluating the rectangular components of the $\bar{m}_{m n}$ vector and comparing them with those of the $\bar{m}_{e / 0 m n}$ given in [7]. Indeed, the rectangular components of $\bar{m}_{m n}$ are easily obtained if one starts by applying (25) for $R_{1}=0$ and $R_{12}=d$. Then:

$$
\bar{m}_{m n}^{(1)}\left(d, \theta_{0}, \phi_{0}\right)=\sum_{\mu=-1,0,1} B_{\mu 1}^{m n}\left(d, \theta_{0}, \phi_{0}\right) \bar{n}_{\mu 1}^{(1)}(0),
$$

a result based on the fact that

$$
\begin{aligned}
& \bar{n}_{-1,1}^{(1)}(0)=-(\hat{x}-i \hat{y}) / 3 ; \quad \bar{n}_{01}^{(1)}(0)=2 \hat{z} / 3 ; \quad \tilde{n}_{11}^{(1)}(0)=2(\hat{x}+i \hat{y}) / 3 \\
& \left.\bar{m}_{\mu s}^{(1)}(0)=0(\text { all } \mu, s) ; \quad \bar{n}_{\mu s}^{(1)}(0)=0 \text { (unless } s=1, \mu=-1,0,1\right) .
\end{aligned}
$$

These results follow easily from (4), (5) and the well known relations among spherical $(\hat{r}, \hat{\theta}$, $\hat{\phi})$ and rectangular $(\hat{x}, \hat{y}, \hat{z})$ unit vectors. Substitution of (A.9) into (A.8) yields the rectangular components of $\bar{m}_{m n}^{(1)}$ in terıns of the symbol $B_{\mu 1}^{m n}(\mu=-1,0,1)$. This, as explained above, verifies the necessity of the sign correction in (30) and (32).

\section{REFERENCES}

[1] J. D. Kanellopoulos and J. G. Fikioris, Acoustic resonant frequencies in an eccentric spherical cavity, J. Acoust. Soc. Am. 64, 286-297 (1978)

[2] C. Müller, Foundations of the mathematical theory of electromagnetic waves, Springer-Verlag, Berlin (English translation), 1969

[3] H. Hönl, A. W. Maue and K. Westpfahl, Theory of diffraction, Handbuch der Physik 25/1, pp. $218-573$. Springer-Verlag, Berlin, 1961

[4] J. Van Bladel, Electromagnetic fields, McGraw-Hill Book Co., New York, N. Y., 1964

[5] O. D. Kellogg, Foundations of potential theory, Dover Publ., New York, N. Y., 1953

[6] O. R. Cruzan, Translational addition theorems for spherical vector wave functions, Quart. Appl. Math. 20, 33-40 (1962)

[7] P. M. Morse and H. Feshbach, Methods of theoretical physics, McGraw-Hill Book Co., New York, N. Y.. 1953

[8] J. A. Stratton, Electromagnetic theory, McGraw-Hill Book Co., New York, N. Y., 1941

[9] H. Y. Yee and N. F. Audeh, Cutoff frequencies of eccentric waveguides, IEEE Trans. on MTT 14, 487-493 (1966) 